\title{
Conduta Obstétrica na Apresentação Pélvica
}

\author{
Obstetric Management in Breech Presentation
}

Márcia Maria Auxiliadora de Aquino, Gabriela Mauro Garcia, Tayse Magally Costa Rodrigues Maria Rita Souza Mesquita, José Guilherme Cecatti, Coríntio Mariani Neto

\section{RESUMO}

\begin{abstract}
Objetivo: avaliar os resultados da assistência ao parto de gestantes com apresentação pélvica. Pacientes e Métodos: estudo descritivo em que foram analisadas 160 gestantes com feto em apresentação pélvica, com recém-nascidos vivos, divididas em 2 grupos conforme o tipo de parto. Foram estudados dados clínicos referentes ao trabalho de parto, parto e recém-nascido (RN). Para os procedimentos estatísticos comparararam-se os partos vaginais às cesarianas, utilizando cálculo de médias, desvio-padrão, $\chi^{2}$ e os testes de Mann-Whitney e te Student. Resultados: o indice geral de cesárea foi de $81,2 \%$. A idade gestacional e o peso do RN foram significativamente menores no grupo de parto vaginal. A prematuridade e o baixo peso do RN estiveram significativamente associados ao parto vaginal. Somente 14 recém-nascidos tiveram indice de Apgar $<7$ ao quinto minuto, quase $60 \%$ deles no grupo de parto vaginal.

Conclusões: esta população teve elevadas taxas de cesárea, com maior morbidade perinatal, prematuridade e baixo peso no grupo com parto vaginal. Tais achados não permitem conclusões acerca da real associação entre apresentação pélvica, via de parto e resultados perinatais, sendo necessário o controle pela idade gestacional e paridade, além de uma decisão aleatória sobre a via de parto.
\end{abstract}

PALAVRAS-CHAVE: Apresentação pélvica. Trabalho de parto. Resultados perinatais. Prematuridade.

\section{Introdução}

O parto pélvico por via vaginal é um parto de dificuldades crescentes, exigindo que na sua assistência estejam presentes um obstetra experiente, anestesista e neonatologista e que as parturientes sejam bem selecionadas quando se opta pela via vaginal.

Na seleção das parturientes para a tentativa de se conseguir um parto vaginal, muitos fatores devem ser levados em conta, incluindo a paridade (a nuliparidade não é um fator contraindicador, mas a multiparidade é um fator facilitador), a bacia óssea materna (que não deve oferecer resistência, ou seja, não deve ter estreitamentos), a atividade uterina (que deve ser adequada, preferencialmente espontânea), a

Hospital Maternidade Leonor Mendes de Barros

Departamento de Tocoginecologia, CAISM/FCM/UNICAMP

Correspondência:

José Guilherme Cecatti

Caixa Postal 6030

13081-970 - Campinas - SP

Fax: (19) 788-9304 prensa abdominal (adequada), a avaliação do feto (quanto à idade gestacional, peso, grau de flexão e rotação da cabeça e variedade de apresentação), estado das membranas ovulares, presença ou não de cicatriz uterina e presença de circulares de cordão ${ }^{1}$.

Entretanto, nas situações clínicas em que não são adequadamente avaliados todos estes parâmetros, nas mulheres em trabalho de parto e com apresentação pélvica, é maior o risco de ocorrência de óbito fetal anteparto, prolapso de cordão umbilical, traumas, baixos índices de Apgar ao nascimento e maior a incidência de morte neonatal quando por via vaginal ${ }^{1}$. Tais resultados são mais freqüentemente resultantes de anóxia fetal intraparto por compressão de cordão, demora acentuada do período expulsivo, traumas obstétricos e malformações fetais. Provavelmente estes fatores estejam relacionados à popularidade que a indicação de cesariana para a resolução de um parto pélvico passou a ter em muitos lugares, sendo quase sistematicamente realizada nestas situações, embora não existam 
ainda conclusões definitivas sobre a melhor via de parto para esta apresentação.

Estudo mexicano realizado em $1997^{2}$, por exemplo, utilizando critérios rigorosos para realização de parto pélvico vaginal, mostrou que o parto vaginal, quando bem selecionado, não mostra diferença significativa quanto à mortalidade perinatal e diminui a morbidade materna, quando comparado com o parto cesáreo. Neste estudo, as principais indicações de cesárea foram deflexão da cabeça e cesárea prévia.

Em 1994 realizou-se no Canadá um "workshop" com o objetivo de desenvolver um protocolo para critérios de seleção de mulheres com apresentação pélvica no termo, para se planejar um parto vaginal e se fazer seguimento intraparto das mulheres em que esta via de parto pode ser considerada ${ }^{3}$. Secundariamente, esta reunião planejou a realização de um ensaio clínico randomizado incluindo mulheres com apresentação pélvica no termo, comparando cesárea eletiva com parto vaginal em mulheres selecionadas.

Os resultados deste consenso mostraram que paridade e idade das mulheres não devem ser critérios de exclusão para parto vaginal. Por outro lado, hiperextensão da cabeça e peso fetal estimado superior ou igual a $4.000 \mathrm{~g}$ devem ser critérios para a realização de parto cesárea. Quanto à maneira de assistir ao parto pélvico vaginal, concluíram que a apresentação pélvica não é contra-indicação para a indução do trabalho de parto; que a condução do trabalho de parto com ocitocina para corrigir a atividade uterina inadequada é razoável, desde que não haja desproporção cefalopélvica; que a indicação de rotura das membranas deve seguir os mesmos critérios da apresentação cefálica quando se trata de apresentação pélvica completa; que o período expulsivo superior a uma hora é indicação de cesárea; que o encravamento de cabeça derradeira deve ser resolvido com fórcipe ou manobra de Mauriceau; e que a versão cefálica externa deve ser realizada apenas a partir da $37^{\mathrm{a}}$ semana $^{3}$.

Este consenso reconheceu ainda que o treinamento profissional dos obstetras é bastante heterogêneo, mostrando o valor de consultar um colega mais experiente na assistência ao parto pélvico vaginal. Um questionário aplicado por um hospital irlandês no seu quadro de pessoal jovem mostrou que somente $39 \%$ dos entrevistados sentiam-se bem treinados pelo serviço para realização de parto pélvico vaginal ${ }^{4}$.

Lindqvist et al. ${ }^{5}$ estudaram 6.542 gestações únicas em apresentação pélvica, encontrando mortalidade perinatal de 0,05\% (2/4.029) e de
$0,09 \%(2 / 2.248)$, respectivamente para parto vaginal e cesárea, sem diferença estatisticamente significativa. O mesmo ocorreu em estudo suíço que avaliou a mortalidade perinatal em $98 \mathrm{mu}-$ lheres com gestação pré-termo, entre 26 e 36 semanas, com gestação única em apresentação pélvica. Ficou demostrado que a cesárea não ofereceu vantagens ao prematuro pélvico ${ }^{6}$.

No entanto, outros estudos mostram diferença significativa na mortalidade perinatal entre parto vaginal e cesárea. Estudo realizado por Erkaya et al. ${ }^{7}$, avaliando 1.040 apresentações pélvicas, encontrou índices de 8,5/1.000 para o parto cesáreo e de 57,9/1.000 para o vaginal. Concluíram que há necessidade de rigorosa seleção de mulheres e treinamento dos obstetras para balancear a morbimortalidade fetal do parto vaginal com o custo e a morbidade materna da cesárea.

Persistindo, portanto, dúvidas ainda quanto à melhor via para a assistência ao parto em apresentação pélvica, o objetivo deste estudo foi avaliar retrospectivamente a assistência ao parto pélvico realizada em uma maternidade pública da cidade de São Paulo, com os resultados comparados quanto à via de parto.

\section{Pacientes e Métodos}

Este foi um estudo clínico descritivo no qual se avaliaram 160 gestantes com diagnóstico de apresentação pélvica e trabalho de parto, que foram internadas no Hospital e Maternidade Leonor Mendes de Barros, uma maternidade pública que atende uma população carente da zona leste da cidade de São Paulo, e que deram à luz na instituição no período de junho de 1998 a junho de 1999. Estas mulheres foram divididas em dois grupos, segundo o tipo de parto (vaginal ou cesáreo).

Para a coleta dos dados utilizou-se um formulário padronizado em que constavam informações sobre as características sociodemográficas e gestacionais das mulheres, dados clínicos referentes ao trabalho de parto, parto e recémnascido. Estes instrumentos foram revisados quanto à qualidade e legitimidade das informações, e os dados codificados e armazenados em arquivo de computador, após testes de consistência. Para análise dos dados utilizaram-se os procedimentos estatísticos de cálculo de média e desvio-padrão, além do $\chi^{2}$, testes $t$ de Student e Mann-Whitney, para a comparação dos grupos segundo a via de parto. O pacote estatístico utilizado foi o Epi.Info 6.02. 


\section{Resultados}

Das 160 gestantes com apresentação pélvica estudadas, 130 tiveram parto por cesárea, o que corresponde a $81 \%$. Não houve diferença significativa entre os grupos quanto à idade mater- na, número de gestações e paridade. As mulheres eram jovens, com média de idade em torno de 26 anos, estando pelo menos em sua segunda gestação e já com um parto anterior. Entretanto, a idade gestacional e o peso dos recémnascidos foram significativamente menores no grupo que teve parto vaginal (Tabela 1).

Tabela 1 - Valores médios de algumas variáveis demográficas e obstétricas das gestantes com apresentação pélvica, segundo a via de parto.

\begin{tabular}{lcccc}
\hline Variáveis & $\begin{array}{c}\text { Total } \\
\mathbf{x} \pm \mathrm{DP}\end{array}$ & $\begin{array}{c}\text { Cesárea } \\
\mathbf{x} \pm \mathbf{D P}\end{array}$ & $\begin{array}{c}\text { Parto Vaginal } \\
\mathbf{x} \pm \mathbf{D P}\end{array}$ \\
\hline Idade & $26.3 \pm 7.3$ & $26.6 \pm 7.4$ & $25.2 \pm 7.1$ & $0.34^{*}$ \\
IG & $37.5 \pm 3.2$ & $37.9 \pm 2.8$ & $35.4 \pm 4.0$ & $0.001^{*}$ \\
Gesta & $2.6 \pm 2.2$ & $2.5 \pm 2.0$ & $3.1 \pm 2.9$ & $0.62^{* *}$ \\
Para & $1.5 \pm 3.5$ & $1.4 \pm 3.8$ & $1.8 \pm 2.3$ & $0.29^{* *}$ \\
Peso RN (g) & $2.818 \pm 671$ & $2894 \pm 645$ & $2.486 \pm 695$ & $0.002^{*}$ \\
N & 160 & 130 & 30 \\
\hline
\end{tabular}

* Teste $t$ de Student

** Teste de Mann-Whitney

Em relação aos fatores associados à apresentação pélvica, os principais foram: nuliparidade, prematuridade, peso do $\mathrm{RN}<2.500 \mathrm{~g}$, peso RN $>3.500$ g, seguidos por multiparidade, placenta prévia e oligoâmnio. Quando as mulheres foram analisadas segundo o tipo de parto, observou-se que apenas prematuridade e peso do $\mathrm{RN}<2.500 \mathrm{~g}$ foram proporcionalmente mais freqüentes no grupo do parto vaginal, o que foi estatisticamente significativo (Tabela 2).

Tabela 2 - Distribuição porcentual de possíveis fatores associados à apresentação pélvica segundo a via de parto.

\begin{tabular}{lccc}
\hline Fatores & Cesárea & Parto vaginal & $\mathbf{p}^{*}$ \\
\hline Prematuridade & 30,0 & 60,0 & 0.003 \\
Nuliparidade & 46,9 & 40,0 & 0.62 \\
Peso RN $<2.500 \mathrm{~g}$ & 27,6 & 50,0 & 0.03 \\
Peso RN $>3.500 \mathrm{~g}$ & 19,2 & 10,0 & 0.35 \\
Total & 130 & 30 & \\
\hline
\end{tabular}

* Teste do $\chi^{2}$

Em cerca de $82 \%$ das mulheres o parto ocorreu por via abdominal, sendo que as suas principais indicações foram: nuliparidade (39 casos), prematuridade (27 casos), cesárea prévia (19 casos) e placenta prévia (7 casos). Cerca de $10 \%$ dos recém-nascidos apresentaram indice de Apgar no quinto minuto menor do que 7 , mais da metade no grupo com parto vaginal, o que foi estatisticamente significativo. Cerca de dois terços dos RN que necessitaram cuidados intensivos neonatais eram do grupo com parto vaginal, o que também foi significativo (Tabela 3). Entre todos os recém-nascidos, 5 tiveram óbito no periodo neonatal, três deles por prematuridade extrema e dois por infecção.

Tabela 3 - Distribuição porcentual dos resultados perinatais em gestantes com apresentação pélvica, segundo a via de parto.

\begin{tabular}{lccc}
\hline Resultados perinatais & Cesárea & Parto vaginal & $\mathbf{p}^{*}$ \\
\hline Apgar $1^{\circ}$ min $<7$ & 25,4 & 43,3 & 0.08 \\
Apgar $5^{\circ}$ min $<7$ & 6,2 & 20,0 & 0.03 \\
Cuidados intensivos & 4,6 & 43,3 & $<0.0000$ \\
Total & 130 & 30 & \\
\hline
\end{tabular}

${ }^{*}$ Teste do $\chi^{2}$

\section{Discussão}

Este estudo retrospectivo mostrou que nesta população de mulheres com diagnóstico de apresentação pélvica, houve predomínio de indicações de parto cesárea em relação ao parto vaginal. As principais indicações foram nuliparidade, prematuridade, cesárea prévia e placenta prévia. Mostrou ainda que cerca de $10 \%$ dos recém-nascidos nasceram deprimidos, avaliação realizada pelo índice de Apgar ao quinto minuto, mais da metade no grupo com parto vaginal, no qual também foi significativamente maior a ne- 
cessidade de cuidados intensivos.

Nesta população, os principais fatores que se mostraram associados à apresentação pélvica foram a nuliparidade, a prematuridade e o baixo peso do recém-nascido, o que já era esperado. Na etiologia da apresentação pélvica incluem-se vícios de conformação do útero, mioma, prenhez múltipla, oligo ou polidrâmnio, malformação fetal, vício pélvico, multiparidade, placenta cornual fúndica e prematuridade ${ }^{8}$. A nuliparidade, mais que um fator associado à apresentação pélvica, constitui, em nossa prática de obstetrícia clínica, a principal indicação ao parto pélvico, pelo temor de complicações no parto vaginal em uma pelve nunca antes testada.

A diferença estatisticamente significativa entre os grupos estudados com relação à prematuridade e baixo peso do recém-nascido mostra não existir uma adequada seleção de casos para o parto vaginal. Tradicionalmente a prematuridade associada à apresentação pélvica é uma indicação para parto cesáreo, pelo proporcionalmente maior volume do pólo cefálico em relação ao pélvico em idades gestacionais precoces. O limite de idade gestacional ou de peso fetal estimado em que a cesárea teoricamente traria beneficios em termos de saúde perinatal é bastante discutivel e controverso. É conhecida, entretanto, a tendência de se permitir parto vaginal em fetos pélvicos muito pequenos e/ou muito prematuros.

A alta incidência de cesárea neste estudo está de acordo com estudos já publicados, especialmente em países em desenvolvimento e na América Latina. Estudo mexicano, por exemplo, encontrou uma taxa de $75 \%$ de partos abdominais em 104 mulheres com diagnóstico de apresentação pélvica ${ }^{2}$. Sharma et $a^{4}{ }^{4}$, por sua vez, concluíram que a alta taxa de partos por cesárea é decorrente também de um inadequado treinamento do quadro de pessoal jovem para o parto vaginal pélvico.

Além disto, esta incidência de parto abdominal poderia diminuir se os serviços adotassem a conduta de tentar realizar a versão cefálica externa depois da $37^{\text {a }}$ semana de gestação. Revisão sistemática mostrou que a versão cefálica externa no termo diminui significativamente a incidência de apresentação não-cefálica ao nascimento e de partos cesáreos ${ }^{9}$. Quando há falha neste procedimento e não há contra-indicação, deve-se optar por parto vaginal; daí a necessidade do treinamento dos profissionais. Sabe-se, entretanto, que poucos serviços de obstetrícia no Brasil têm pessoal adequadamente treinado e capacitado para qualquer um destes procedimentos.
Quanto às indicações de cesárea encontradas neste estudo, pode-se dizer que estão de acordo com a literatura médica. Estudo escocês mostrou que as principais indicações de cesárea em mulheres com apresentação pélvica foram a nuliparidade, cesárea prévia e multiparidade ${ }^{10}$. Não existem, contudo, evidências científicas concretas que suportem esta conduta.

Em relação aos baixos índices de Apgar no primeiro minuto encontrados neste estudo, é conhecida esta associação com apresentação pélvica, em decorrência da necessidade, em muitos casos, de utilização de manobras para auxiliar na expulsão do feto ${ }^{8}$. Já o pior resultado perinatal, com baixo índice de Apgar no $5^{\circ} \mathrm{mi}-$ nuto para o grupo do parto vaginal, sugere existir uma inadequada seleção das mulheres para esta via, a possibilidade de diferenças de treinamento entre os profissionais, além da própria prematuridade, e a possibilidade real de que esta via não seja a melhor para o parto pélvico. Isto é possível em instituições de ensino e treinamento, com residência médica e com preceptores de diferentes idades, com distintos tempos de treinamento e experiência.

Apesar deste estudo descritivo provavelmente ter indicado a necessidade de maior treinamento para o parto pélvico vaginal e de se estimular a tentativa da versão cefálica externa na instituição, algumas limitações metodológicas devem ser realçadas, para limitar ou relativizar as implicações de seus achados. Tais limitações dizem respeito ao controle pela via de parto, idade gestacional e paridade, além da possivel comparação com grupo semelhante de partos em apresentação cefálica, ou a decisão aleatória sobre o tipo de parto para a apresentação pélvica, para poder se concluir sobre a associação entre apresentação pélvica, via de parto e resultados perinatais.

Estudos comparativos, prospectivos e randomizados, inclusive com subgrupos de análise (por exemplo, na situação de cicatriz de cesárea) são necessários para melhor se avaliar a morbidade materna e a morbimortalidade perinatal de acordo com a via de parto em mulheres com apresentação pélvica, excluindo os fatores confundidores e com tamanho de amostra adequado.

Uma revisão sistemática atualizada concluiu que ainda não há evidências suficientes sobre a melhor via de parto na apresentação pélvica. Refere ainda que um estudo multicêntrico randomizado, em realização sob a coordenação de um grupo canadense, mas com participação de clínicas inclusive no Brasil, poderá concluir definitivamente sobre qual é a melhor interven- 
ção nesta condição ${ }^{11}$. Mais recentemente, em abril de 2000, foi interrompida a admissão de novos casos para este estudo multicêntrico, pela evidência de resultados claramente favoráveis ao parto por cesárea planejada na apresentação pélvica ${ }^{12}$. Tais resultados poderão no futuro fundamentar uma mudança de orientação completa na assistência obstétrica aos partos com apresentação pélvica.

\section{SUMMARY}

Purpose: to evaluate the results of assistance to breech deliveries.

Methods: this was a descriptive study where 160 pregnant women with breech presentation and live newborns were analyzed. They were divided into two groups according to the route of delivery. Clinical data concerning labor, delivery and newborns were studied. For statistical analysis vaginal deliveries were compared with cesarean sections using mean and standard deviation estimates, Student's t, Mann-Whitney and $\chi^{2}$ tests.

Results: the global cesarean section rate was $81.2 \%$. The gestational age and the weight of the newborns were significantly lower in the vaginal delivery group. Prematurity and low birth weight were significantly associated with vaginal delivery. Only 14 newborns had an Apgar score below 7 at the fifth minute, almost $60 \%$ of them in the vaginal delivery group.

Conclusions: this population presented a high cesarean section rate and also high perinatal morbidity, prematurity and low birth weight in the vaginal delivery group. These findings do not allow conclusions regarding the real relationships among breech presentation, route of delivery and perinatal outcomes. The control regarding gestational age and parity, besides a random decision on the route of delivery, is necessary for future conclusions.

KEY WORDS: Breech presentation. Labor. Perinatal results. Prematurity.

\section{Referências}

1. Cunningham FG, MacDonald PC, Gant NF, Leveno KJ, Gilstrap LC $3^{\text {rd }}$ Dystocia due to abnormalities in presentation, position or development of the fetus. In: Williams Obstetrics. $19^{\text {th }}$ ed. East Norwalk: Appleton \& Lange; 1993. p.493-520.
2. Neri Ruiz ES, Valerio Castro E, Cardenas Arias R, Navarro Milla C. Presentación pelvica, siempre cesarea? Ginecol Obstet Mex 1997; 65:474-7.

3. Hannah WJ. The Canadian Consensus on breech management at term. J Soc Obstet Gynecol Can 1994; 16:1839-48.

4. Sharma JB, Newman MR, Boutchier JE, Williams A. National audit on the practice and training in breech deliveries in the United Kingdom. Int J Gynaecol Obstet 1997; 59:103-8.

5. Lindqvist A, Norden-Lindeberg S, Hanson U. Perinatal mortality and route of delivery in term breech presentations. Br J Obstet Gynaecol 1997; 104:1288-91.

6. Ziadeh S, Abu-Heija AT, El-Sunna E, El-Jallad MF, Shatnawi A, Obeidat A. Preterm singleton breech in North Jordan: vaginal versus abdominal delivery. Gynecol Obstet Invest 1997; 44:16972 .

7. Erkaya S, Tuncer RA, Kutlar I, Onat N, Ercakmak S. Outcome of 1040 consecutive breech deliveries: clinical experience of a maternity hospital in Turkey. Int J Gynaecol Obstet 1997; 59:115-8.

8. Neme B. Distócia fetal. In: Neme B, editor. Obstetrícia Básica. São Paulo: Sarvier; 1994. p.508-37.

9. Hofmeyer GJ. External cephalic version at term. Cochrane Review. In: The Cochrane Library; Issue 4, 1997. Oxford: Update Software.

10.Wilkinson C, McIlwaine G, Bouton-Jones C, Cole S. Is a rising caesarean section rate inevitable? Br J Obstet Gynaecol 1998; 105:45-52.

11.Hofmeyer GJ, Hannah ME. Planned caesarean section for term breech delivery (Cochrane Review). In: The Cochrane Library, Issue 4, 1999. Oxford: Update Software.

12. University of Toronto. Maternal, Infant and Reproductive Health Research Unit. Term Breech Trial News [serial online] 2000; 6(4). Available from: URL: http://www.utoronto.ca/ miru/breech 